\title{
Experimental micrometer-displacement measurements based on optical vortices
}

\section{Medidas experimentales de desplazamientos micrométricos basadas en vórtices ópticos}

\author{
N. Londoño ${ }^{1 *}$, E. Rueda ${ }^{1}$, J. A. Gómez ${ }^{2}$. D. Amaya ${ }^{3}$, A. Lencina ${ }^{4}$ \\ 1. Grupo de Óptica y Fotónica, Instituto de Física, Universidad de Antioquia U de A, Calle 70 No. 52-21, \\ Medellín, Colombia. \\ 2. Grupo de Física Básica y Aplicada. Politécnico Colombiano Jaime Isaza Cadavid. Medellín-Colombia. \\ 3. Centro de Investigaciones Ópticas, CONICET-UNLP-CIC, P.O. Box 3, 1897 Gonnet, Argentina. \\ 4. Laboratorio de Análisis de Suelos, Facultad de Agronomía, Universidad Nacional del Centro de la Provincia \\ de Buenos Aires, CONICET, P. O. Box 47, 7300 Azul, Argentina. \\ (*) E-mail: natalia.londonol@udea.edu.co
}

Received: 27/10/2016 Accepted: 10/04/2017

DOI: $10.7149 /$ OPA.50.2.49018

\begin{abstract}
:
In this work, a system for measuring micrometer-displacements based on the characteristics of optical vortices is presented. In the proposal, a binary vortex-producing lens (BVPL) programmed to generate optimized optical vortices is transversally displaced from the optical axis, inducing perturbations on the optical characteristics of the vortices that are used as transduction parameters. Specifically, the method proposed theoretically by Anzolin et al. [18], which is based on the asymmetry of the intensity patterns of the off-axis optical vortices, is studied experimentally by using BVPLs. Experimental implementation is completely described and compared with theoretical results, likewise, metrological characteristics of the experimental metrological system are analyzed. Based on the results, we experimentally confirm the possibility of creating high sensitivity metrological systems by using optical vortices, opening the door for new vortex metrology techniques.
\end{abstract}

Key words: Optical Vortices, Metrology, Micro-displacement.

\section{RESUMEN:}

En este trabajo, un sistema para medir desplazamientos micrométricos basados en las características de vórtices ópticos es presentado. En la propuesta, una lente productora de vórtices binaria (BVPL) programada para generar vórtices ópticos optimizados es desplazada transversalmente del eje óptico, induciendo perturbaciones en las características ópticas de los vórtices que son usadas como parámetros de transducción. Específicamente, el método propuesto por Anzolín et al [18], el cuál es basado en la asimetría de los patrones de intensidad de los vórtices ópticos fuera del eje, es estudiado experimentalmente usando BVPLs. Se describe completamente la implementación experimental y se compara con los resultados teóricos, así mismo, se analizan características metrológicas del sistema metrológico experimental. En base a los resultados, confirmamos experimentalmente la posibilidad de crear sistemas metrológicos de alta sensibilidad utilizando vórtices ópticos, abriendo la puerta para nuevas técnicas de metrología de vórtices.

Palabras clave: Vórtices ópticos, Metrología, micro desplazamientos.

\section{REFERENCES AND LINKS / REFERENCIAS Y ENLACES}

[1] J. F. Nye and M. V. Berry, "Dislocations in wave trains," Proc. R. Soc. London Ser. A 336, 165-190 (1974). https://doi.org/10.1098/rspa.1974.0012 
[2] Mohamed El Ketara and Etienne Brasselet, "Observation of Self-Induced Optical Vortex Precession," Phys. Rev. Lett. 110, 233603 (2013). https://doi.org/10.1103/PhysRevLett.110.233603

[3] J. Leach, M. R. Dennis, J. Courtial1 and M. J. Padgett, “Vortex knots in light," New J. Phys. 7, 55 (2005). https://doi.org/10.1088/1367-2630/7/1/055

[4] R. K. Tyson, M. Scipioni, and J. Viegas, "Generation of an optical vortex with a segmented deformable mirror," Appl. Opt. 47, 6300-6306 (2008). https://doi.org/10.1364/A0.47.006300

[5] S. Fürhapter, A. Jesacher, S. Bernet, and M. Ritsch-Marte, "Spiral phase contrast imaging in microscopy," Opt. Exp. 13, 689-694 (2005). https://doi.org/10.1364/OPEX.13.000689

[6] J.A. Gómez, E. Rueda, Á. Salazar, M. Tebaldi, N. Bolognini, A. Lencina, "Effects of the induced birefringence in photorefractive crystals on speckle optical vortices," Opt. Eng. 50, 359-365 (2012). https://doi.org/10.1016/j.optlaseng.2011.10.021

[7] H. Sun, X. Wang, and P. Sun, "In-plane displacement measurement using optical vortex phase shifting," Appl. Opt. 21, 5610-5613 (2016). https://doi.org/10.1364/A0.55.005610

[8] J. H. Lee, G. Foo, E. G. Johnson, and G. A. Swartzlander Jr, "Experimental Verification of an Optical Vortex Coronagraph," Phys. Rev. Lett. 97, 0539011 (2006). https://doi.org/10.1103/PhysRevLett.97.053901

[9] V. Y. Bazhenov, M. V. Vasnetsov, and M. S. Soskin, "Laser beams with screw dislocations in their wavefronts," JETP Lett. 52, 429-431 (1990).

[10] M. W. Beijersbergen, R. P. C. Coerwinkel, M. Kristensen, and J. P. Woerdman, "Helical-wavefront laser beams produced with a spiral phaseplate," Opt. Commun. 112, 321-327 (1994). https://doi.org/10.1016/0030-4018(94)90638-6

[11] E. Rueda, D. Muñetón, J. A. Gómez, and A. Lencina, "High-quality optical vortex-beam generation by using a multilevel vortex-producing lens," Opt. Lett. 38, 3941-3944 (2013). https://doi.org/10.1364/OL.38.003941

[12] N. Londoño, E. Rueda, J. A. Gómez, and A. Lencina, "Generation of optical vortices by using binary vortex producing lenses," Appl. Opt. 54, 796-801 (2015). https://doi.org/10.1364/A0.54.000796

[13] L. Janicijevic, and S. Topuzoski, "Fresnel and Fraunhofer diffraction of a Gaussian laser beam by forkshaped gratings," J. Opt. Soc. Am. A 25, 2659-2669 (2008). https://doi.org/10.1364/JOSAA.25.002659

[14] L. Angel-Toro, D. Sierra-Sosa, M. Tebaldi, and N. Bolognini, "In-plane displacement measurement in vortex metrology by synthetic network correlation fringes," J. Opt. Soc. Am. A 30, 462-469 (2013) https://doi.org/10.1364/JOSAA.30.000462

[15] A. Dudley, N. Majola, N. Chetty, and A. Forbes, "Implementing digital holograms to create and measure complex-plane optical fields," Am. J. Phys. 84, 106-112 (2016). https://doi.org/10.1119/1.4935354

[16] G. Anzolin, F. Tamburini, A. Bianchini, G. Umbriaco, and C. Barbieri, "Optical vortices with starlight," Astron. Astrophys. 488, 1159-1165 (2008). https://doi.org/10.1051/0004-6361:200810469

[17] F. Tamburini, G. Anzolin, G. Umbriaco, A. Bianchini, and C. Barbieri, “Overcoming the Rayleigh Criterion Limit with Optical Vortices," Phys. Rev. Lett. 97, 163903 (2006). https://doi.org/10.1103/PhysRevLett.97.163903

[18] G. Anzolin, F. Tamburini, A. Bianchini, and C. Barbieri, "Method to measure off-axis displacements based on the analysis of the intensity distribution of a vortex beam," Phys. Rev. A. 79, 033845 (2009). https://doi.org/10.1103/PhysRevA.79.033845

\section{Introduction}

Optical waves can have punctual phase singularities, commonly known as optical vortices (OVs). These OVs are localizations in space were the amplitude of the wave is zero and its phase is undefined [1-3], while in the surroundings the phase takes a helicoidal form, usually represented with the term $\exp (\mathrm{im} \theta)$, where $\theta$ is the azimuthal angle and $\mathrm{m}$ is the topological charge representing the number of $2 \pi$ discontinuities of the wavefront [4]. Due to these features, OVs have been widely used in metrological applications [5, 6], phase shifting interferometry [7], stellar choronography [8], among others. To generate the OVs, computer 
generated holograms (CGHs) [9], spiral phase plates (SPPs) [10] and vortex-producing lenses (VPL)[11] are commonly used. For the case of VPLs, the transmission function of the SPP is multiplied by the transmission function of a Fresnel lens, which allows the generation of high quality optical vortices by using discretized masks of very few levels. In a recent paper we have obtained experimentally high quality OVs, with charges between $m=1$ and $m=5$ by using a binary VPL (BVPL) [12]. The BVPL was displayed in a LC-SLM with a maximum phase modulation of $\theta_{\text {máx }} \approx 1.2 \pi$. Therefore, a Gaussian beam symmetric around the propagation axis diffracted by a BVPL, produces a donut-shape beam which is well described by a Kummer function [13].

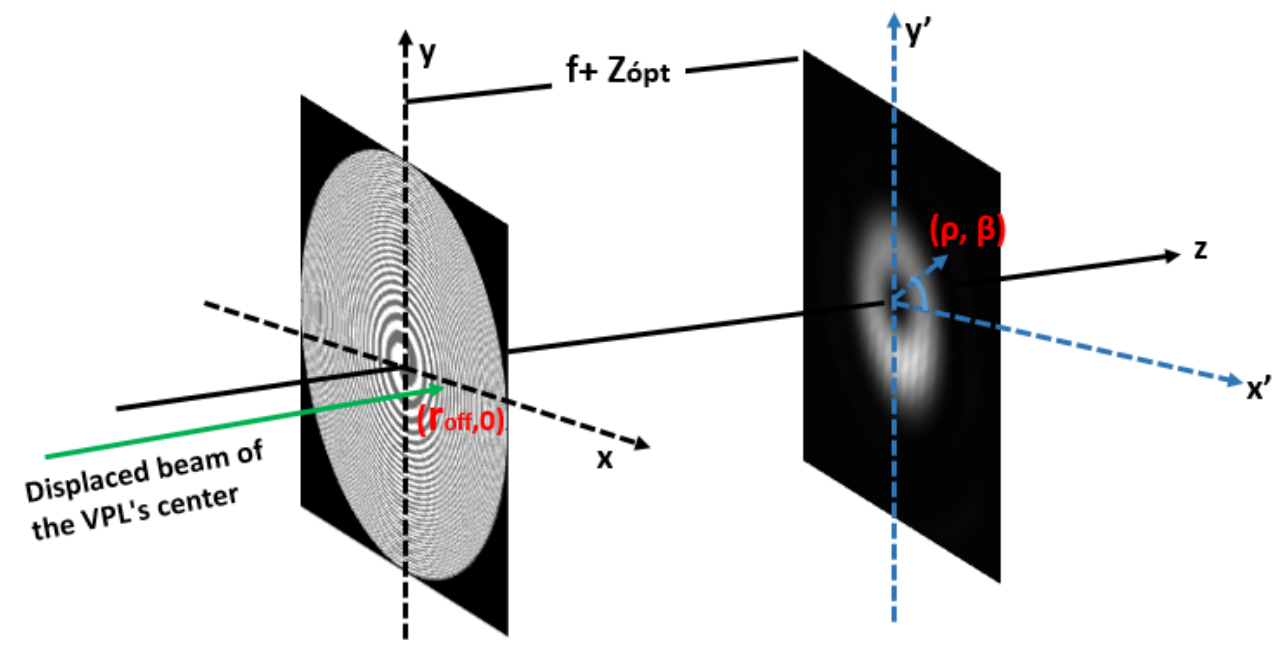

Fig. 1. Vortex producing lens and its corresponding vortex.

Although metrology systems based on the properties of the vortex distribution of speckle patterns have been widely studied [14], more recently an interest in the analysis of the morphological characteristics of Laguerre Gauss and Kummer beams have grown [15]. As can be expected, if a relative movement between the input beam and the center of the VPL occurs, a loss of the symmetry is registered in both, intensity and phase (see Fig. 1). This characteristic has been strongly used in astronomy applications [16, 17]. In particular, Anzolin et al. proposed analytically and numerically a method to measure very small displacements based on the degree of asymmetry of the intensity pattern of an off-axis vortex beam [18]. In this work, we studied experimentally, using a BVPL, the asymmetry of the intensity patterns of the off-axis optical vortices. The experimental setup for the metrological system based on the use of BVPLs, is well described and results of the technique for topological charges from $m=1$ to $m=5$ are presented. Performance of the method is compared with the Anzolin's theoretical model, finding excellent agreement. Results are highly promissory and showed that low efficiency of the BVPLs is not a problem for the implementation of the technique and, instead, use of low cost LC-SLMs represents an advantage in terms of implementation.

\section{Theoretical intensity analysis}

As was mentioned, Anzolin et al. proposed theoretically a method to measure small displacements based on the asymmetry of the intensity pattern of an off-axis optical vortex. In the model, they considered a Gaussian beam impinging perpendicularly on a SPP displaced from the axis, which produces in far field an asymmetric intensity optical vortex. Therefore, if the off-axis position can be written as $\left(\mathrm{r}_{\text {off }}, \theta_{\text {off }}\right)$, the field in the focal plane of a lens located after the SPP, can be expressed as [18]:

$$
u_{m}(\rho, \beta)=c_{0} i^{-m}\left(\frac{\pi^{3} / 2}{2 w_{0}^{2}}\right) e^{i m \psi} e^{-w_{0}^{2} r_{o f f}^{\prime 2}} e^{-\frac{\gamma^{2}}{8 w_{0}^{2}}}\left(\frac{\gamma}{2 w_{0}}\right)\left[I_{(m-1) / 2}\left(\frac{\gamma^{2}}{8 w_{0}^{2}}\right)-I_{(m+1) / 2}\left(\frac{\gamma^{2}}{8 w_{0}^{2}}\right)\right] ;
$$

where $\rho$ and $\beta$ are the radial and azimuthal positions in the observation plane, $w_{0}=\frac{f}{k w}, f$ is the focal length of the lens, $k=\frac{2 \pi}{\lambda}, w$ is the beam waist, $\eta=\frac{\rho}{2 w_{0}}, r^{\prime}{ }_{o f f}=r_{o f f} k / \mathrm{f}$, and $\mathrm{c}_{0}$ is a constant factor. Due to the functional form of the field given in (1), this kind of beam is known as Kummer vortex beam.

In expression (1), the following conditions must be satisfied: 


$$
\left\{\begin{array}{c}
\gamma^{2}=\rho^{2}+4 i w_{0}^{2} r^{\prime}{ }_{o f f} \rho \cos \left(\beta-\theta_{o f f}\right)-4 w_{0}^{4} r^{\prime 2}{ }_{o f f} \\
\tan \psi=\frac{\rho \sin \beta+2 i w_{0}^{2} r_{o f f} \sin \theta_{o f f}}{\rho \cos \beta+2 i w_{0}^{2} r^{\prime}{ }_{o f f} \cos \theta_{o f f}}
\end{array}\right.
$$

The effect of the off-axis incidence is the shift of the phase singularity to the position $(\rho, \beta)=$ $\left(2 w_{0} r^{\prime}{ }_{\text {off }}, \theta_{\text {off }}+\pi / 2\right)$ and, as a result, the intensity distribution of the vortex becomes asymmetric, showing two different peaks in the observation plane (See Figure 2).

a)

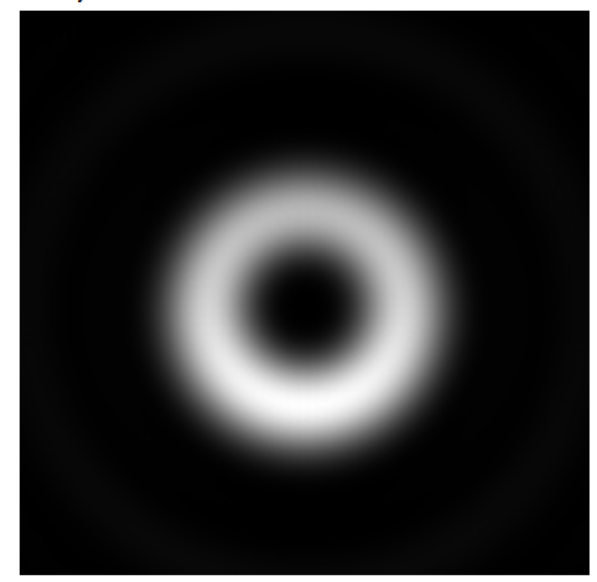

b)

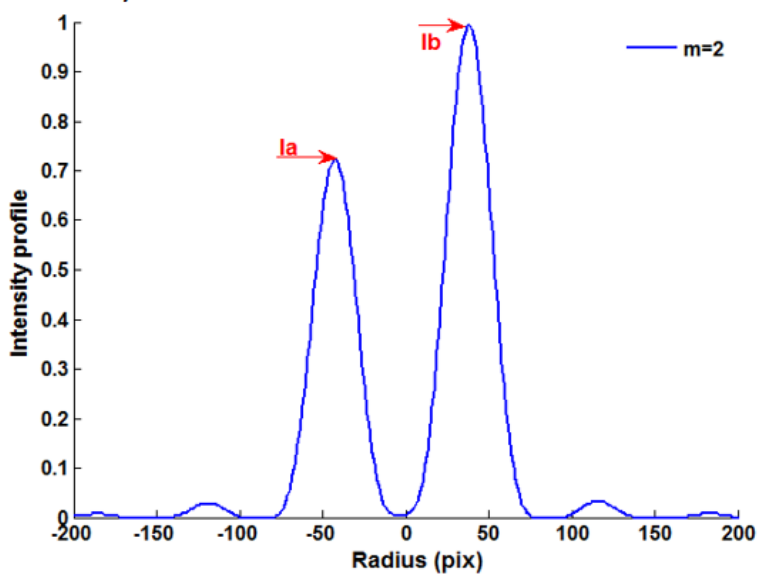

Fig. 2. Simulated off-axis optical vortex $(m=2)$ produced by BVPL. a) Transverse intensity distribution in the observation plane, b) Radial intensity profile.

In this way, to evaluate numerically the asymmetry as a transduction parameter, in the referenced work an intensity ratio $R=I_{a} / I_{b}$ was proposed, where $I_{a}, I_{b}$ corresponds to the lower and higher intensity peaks in the radial intensity profile, respectively. Likewise, the displacement was normalized at the half width of the intensity maximum, therefore, the movement from the axis was measured as $r_{o f f} / a$, where $a=w \sqrt{\ln 2 / 2}$. Using these variables, it is possible to define the parameter $\mathrm{R}$ which decays exponentially as the displacement increases

$$
R=k_{1} e^{-\left(k_{2} r_{o f f}\right) / a}
$$

The constants $k_{1}$ and $k_{2}$ in equation (3) are obtained by best fitting the simulated curves. In Figure 3 , we present our simulations of the behavior of the parameter R, for an off-axis optical vortex with charge $m=1$ to $m=5$ by using BVPL. Results indicate that by using the parameter $R$ it is possible to create a very sensitive displacement measurement system whose sensitivity depends on the topological charge, in agreement with Anzolin et al. simulated results.

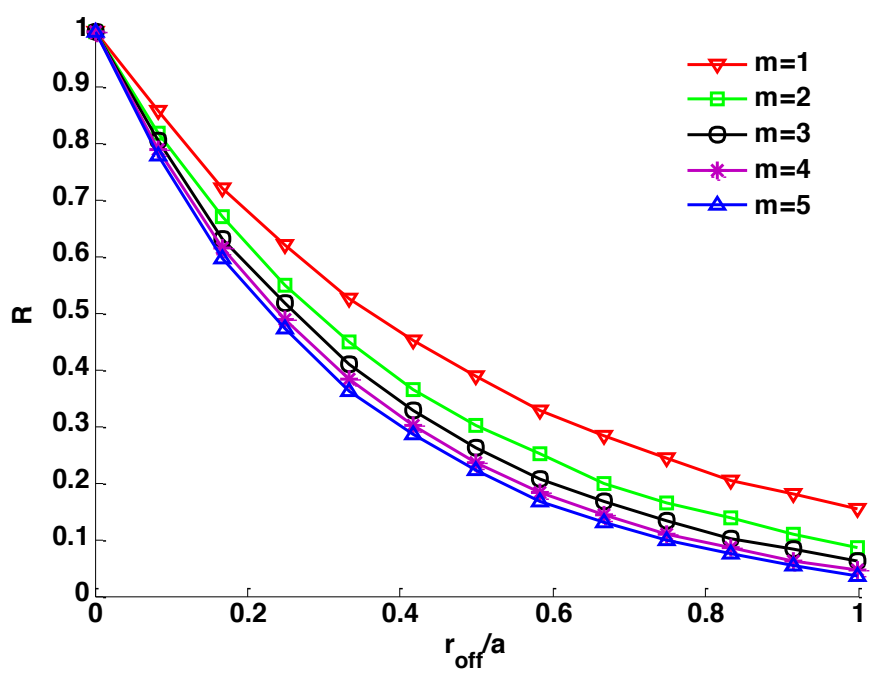

Fig. 3. Theoretical peaks intensity ratio vs normalized off-axis displacement by using BVPL. 


\section{Experimental setup}

In this section, we present the experimental implementation of the metrological system. Unlike the theoretical work, we used as phase mask a vortex-producing lens (VPL), defined by the equation $t(r, \theta)=$ $e^{i m \theta} e^{-i \frac{\pi r^{2}}{\lambda f_{F R}}}$ wich corresponds to the product of a SPP with a Fresnel lens of focal distance $f_{F R}$ designed for a wavelength $\lambda$ [11]. As has been previously demonstrated, the use of VPLs have allowed the generation of high quality optical vortices reducing notably the discretization requirements. In particular, it was possible to construct optical vortices by using only two levels discretized VPLs, i.e, (BVPLs) [12]. In Figure 4, the sequence for the construction of a binary VPL with $m=1$ is presented. Initially, a SPP and a Fresnel lens are generated and superposed forming a VPL of 256 levels and, then, the obtained VPL is discretized according to the possible phase levels; in this case two-phase levels.

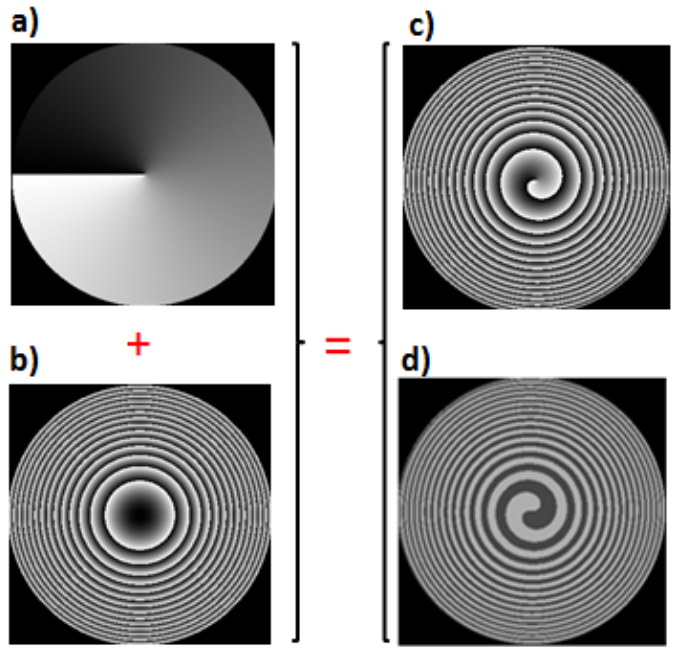

Fig. 4. Construction of binary VPL for $m=1$ a) SPP with $N=256$, b) Fresnel lens with $N=256$ ( $2 \pi$ modulation), c) Superposition of the SPP and the Fresnel lens to create a VPL with N=256 d) two-phase levels discretized VPL (BVPL) [12].

For the generation and characterization of the OVs, the BVPL is displayed in the LC-SLM of the experimental setup presented in Figure 5. There, linear polarizers $\mathrm{P}_{1}, \mathrm{P}_{2}$ and a $\lambda / 4$ waveplate are introduced to guarantee the maximum phase modulation with the lower amplitude modulation. The laser beam is spatially filtered and collimated with the spatial filter 0 and lens $\mathrm{L}_{1}$, respectively, and the beam diameter $(2 w=4.6 \mathrm{~mm})$ is adjusted with diaphragm D. Subsequently, the beam passes through the LC-SLM (RealLight, RL-SLM-T1 with $1024 \times 768$ pixels of $26 \mu \mathrm{m}$ each one) and the light emerging is focalized with a convergent lens $\mathrm{L}_{2}$ located at a distance $f$ (equal to its focal length of $200 \mathrm{~mm}$ ) of the LC-SLM. The OV with topological charge equal to the programmed in the BVPL is generated in the first diffraction order and it is observed at a distance $Z_{\text {ópt }}$ measured from the back focal plane of $\mathrm{L}_{2}$; only at this distance the functional form of the field obtained with the VPL will be equal to that reported by Anzolin et al. using a SPP (eq.(1)). In this case, we have used a diverging Fresnel lens, so $Z_{\text {ópt }}=f^{2} / f_{F R}[10,15]$. The intensity of the $\mathrm{OV}$ at the observation plane is registered by using a CMOS camera coupled to a $20 \mathrm{X}$ microscope objective. By using this arrangement, it is possible to obtain in real time intensity distributions, allowing the implementation of the proposed metrological system.

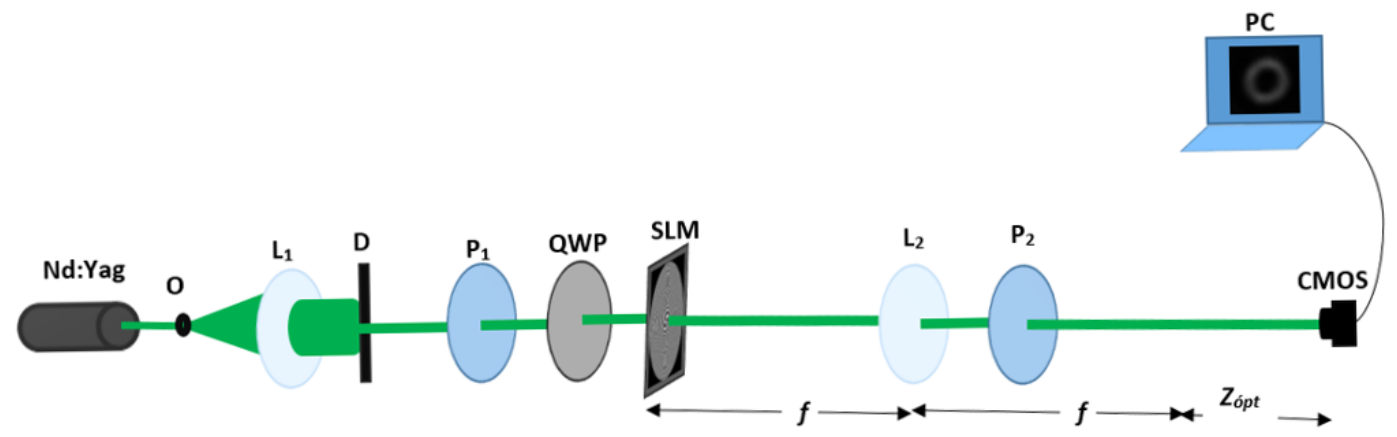

Fig. 5. Experimental setup. 
To experimentally evaluate the behavior of the intensity asymmetry of an OV as a function of the off-axis displacement, the phase mask was directly displaced from the SLM centre. In this case, the displacement steps were integer numbers of $26 \mu \mathrm{m}$ (which is equivalent to one pixel size of the SLM). Likewise, the movements of the VPL were codified only on one axis, therefore $\boldsymbol{r}_{\boldsymbol{o f f}}=\boldsymbol{x}_{\boldsymbol{o f f}}$. Displacements in the interval $\mathbf{0}<\frac{r_{\text {off }}}{\boldsymbol{a}}<\mathbf{0 . 6}$ were set in the SLM and registered by the camera. Upper movements were not registered for experimental limitations. In Figure 6 the experimental displaced-vortices and their respective intensity profiles are presented. Note that for displacement in axis $\boldsymbol{x}$, asymmetries in intensity occur in axis $\boldsymbol{y}$.

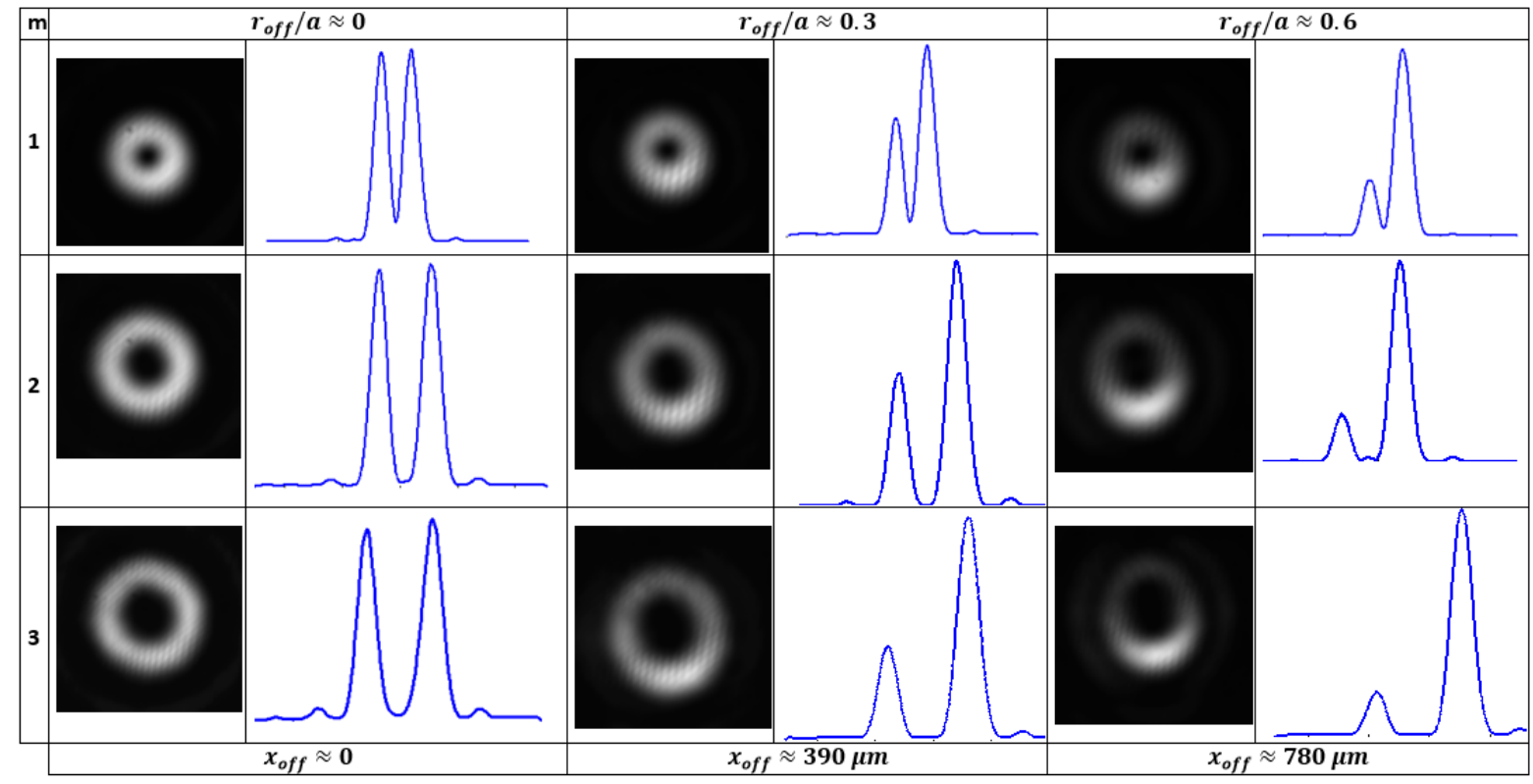

Fig. 6. Experimental displaced vortices and their intensity profiles, for $m=1$ to 3 .

In Figure 7, the experimental behavior of the parameter $\mathrm{R}$ as a function of the off-axis displacement for different topological charges is presented. As can be appreciated, the performance of the parameter $\mathrm{R}$ depends on the topological charge and, moreover, the decreasing rate is stronger for higher topological charges. These elements are in agreement with the theoretical results reported by Anzolin et al.

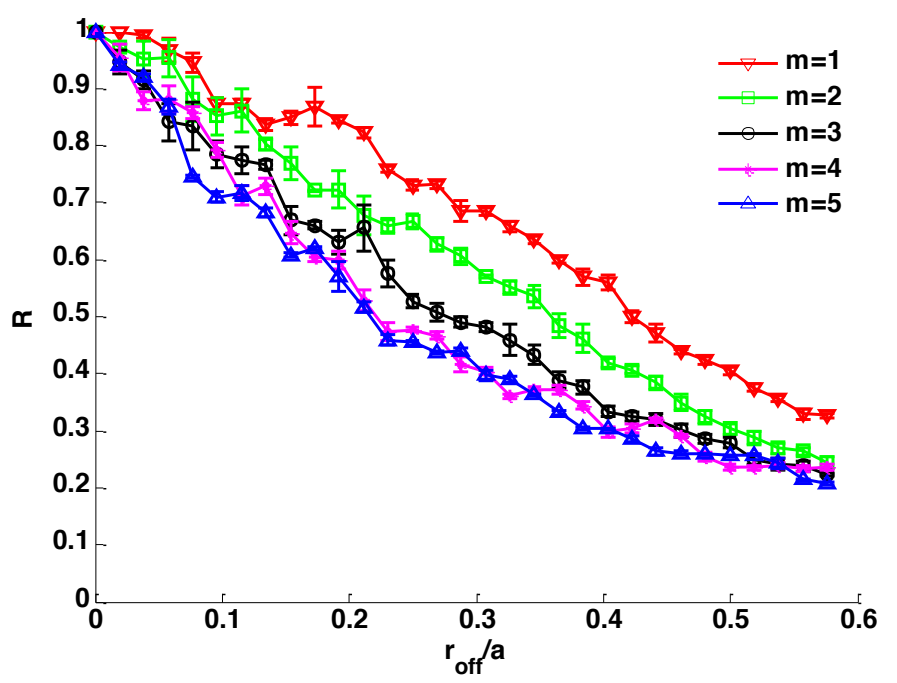

Fig. 7. Experimental peaks intensity ratio $R$ vs normalized off-axis displacement.

In Figure 8, the fitting-curve for the experimental data of $m=3$ is presented. There, it is clear the existence of an exponential behavior. Fitting parameters $k_{1}, k_{2}$ and the correlation coefficient $\left(\mathcal{R}^{2}\right)$, obtained for topological charges $m=1$ to $m=5$, are listed in Table 1. Difference between experimental parameters and computational ones calculated by Anzolin et al. is due to physical characteristics of the setup, however, it is 
clear that the behavior of the parameter $R$ is corroborated. It is important to mention that the error bars for $R$ were calculated by computing the deviation of several measures in each experimental point, with a student factor equivalent to $98 \%$ confidence.

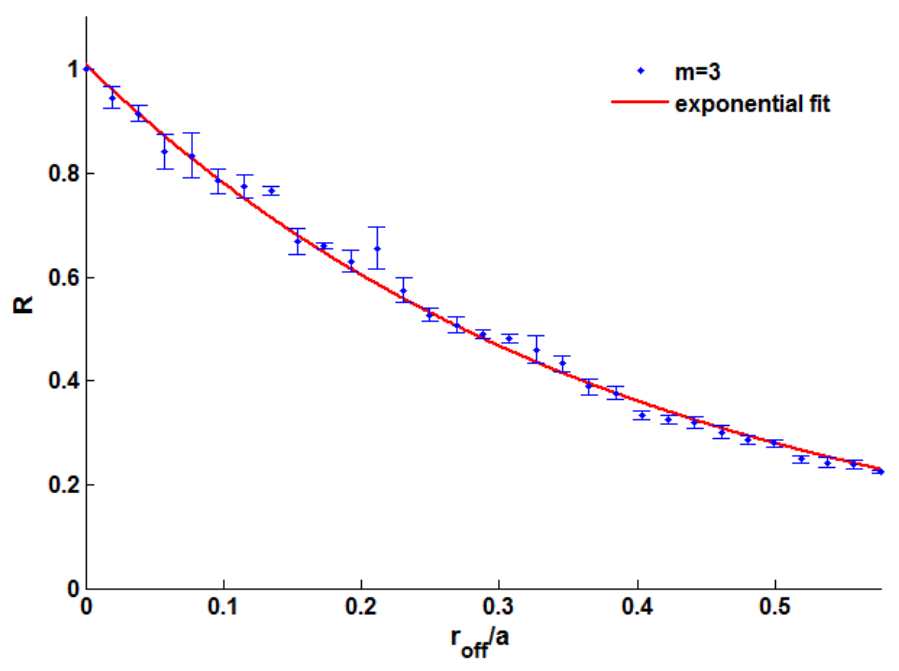

Fig. 8. Best fitting for experimental measures with $\mathrm{m}=3$.

TABLE 1 . Values of $k_{1}$ and $k_{2}$ parameters with $95 \%$ confidence, and the correlation coefficient $\mathcal{R}^{2}$, for $m=1$ to 5 .

\begin{tabular}{|c|c|c|c|}
\hline \hline $\mathbf{m}$ & $\boldsymbol{k}_{\mathbf{1}}$ & $\boldsymbol{k}_{\mathbf{2}}$ & $\mathcal{R}^{2}$ \\
\hline \hline $\mathbf{1}$ & $1.06 \pm 0.04$ & $1.73 \pm 0.15$ & 0.95 \\
$\mathbf{2}$ & $1.02 \pm 0.03$ & $2.14 \pm 0.12$ & 0.98 \\
$\mathbf{3}$ & $1.01 \pm 0.01$ & $2.57 \pm 0.06$ & 0.99 \\
$\mathbf{4}$ & $1.00 \pm 0.02$ & $2.80 \pm 0.09$ & 0.99 \\
$\mathbf{5}$ & $0.99 \pm 0.02$ & $2.90 \pm 0.10$ & 0.99 \\
\hline \hline
\end{tabular}

Based on these empirical parameters and by using equation (3) it is possible to estimate the sensitivity of the system as [18]:

$$
\frac{r_{o f f}}{a} \geq \frac{1}{k_{2}} \ln \left(\frac{k_{1}}{R}\right)
$$

From expression (4) it is clear that the sensitivity of this metrological system is not constant and, instead, it depends on the parameter $R$, the beam waist $w(a=w \sqrt{\ln 2 / 2})$ and the topological charge $m$. In Table 2 some calculations of the displacement sensitivity are presented.

TABLE 2. Sensitivity of the metrological system to minimum detectable displacement $\left(\Delta r_{o f f}\right)$, as a function of the parameter " $a$ ".

\begin{tabular}{|c|c|c|c|c|c|}
\hline $\boldsymbol{R}$ & & & & & \\
\hline \hline $\mathbf{0 . 9 8}$ & $0.045 a$ & $0.020 a$ & $0.011 a$ & $0.007 a$ & $0.002 a$ \\
$\mathbf{0 . 8 1}$ & $0.156 a$ & $0.109 a$ & $0.086 a$ & $0.075 a$ & $0.069 a$ \\
$\mathbf{0 . 4 4}$ & $0.509 a$ & $0.394 a$ & $0.323 a$ & $0.293 a$ & $0.281 a$ \\
\hline \hline
\end{tabular}

From Table 2, it is possible to appreciate that $R$ values close to one produce higher sensitivities, likewise, as the parameter $R$ decreases, the sensitivity decreases too. Additionally, the sensitivity of the metrological system can be tuned modifying the topological charge and the beam waist.

\section{Conclusions}

In this work, the possibility of experimentally implementing a micrometer-displacement measurement system based on OVs created by using discrete phase masks of only two-levels (BVPL) was demonstrated. The strategy was supported on the theoretical results proposed by Anzolin et al. and, therefore, was implemented by measuring intensity asymmetries of off-axis OVs. BVPLs with topological charges $\mathrm{m}=1$ to 
$\mathrm{m}=5$ were displayed on a low-cost LC-SLM with a maximum phase modulation $\theta_{\text {máx }} \approx 1.2 \pi$. Displacements were induced directly in the modulator. Experimental results were in agreement with the theoretical predictions. Finally, it was found that two experimental parameters, topological charge $\mathrm{m}$ and the beam waist $w$, can act as tuners for this type of systems.

\section{Acknowledgements}

Universidad de Antioquia (2015-760) - Estrategia de sostenibilidad 2014-2015; Politécnico Colombiano Jaime Isaza Cadavid. 